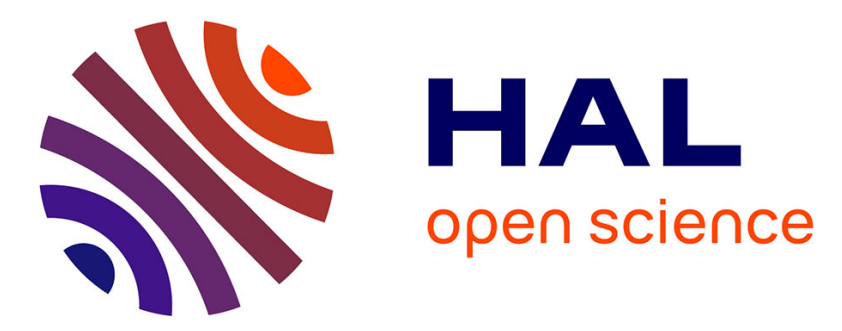

\title{
Conformal Propagation and Near-Omnidirectional Radiation with Surface Plasmonic Clothing
}

\author{
Xi Tian, Qihang Zeng, Denys Nikolayev, John S. Ho
}

\section{To cite this version:}

Xi Tian, Qihang Zeng, Denys Nikolayev, John S. Ho. Conformal Propagation and NearOmnidirectional Radiation with Surface Plasmonic Clothing. IEEE Transactions on Antennas and Propagation, 2020, 68 (11), pp.7309-7319. 10.1109/TAP.2020.2998216 . hal-02948496

\section{HAL Id: hal-02948496 https://hal-univ-rennes1.archives-ouvertes.fr/hal-02948496}

Submitted on 25 Sep 2020

HAL is a multi-disciplinary open access archive for the deposit and dissemination of scientific research documents, whether they are published or not. The documents may come from teaching and research institutions in France or abroad, or from public or private research centers.
L'archive ouverte pluridisciplinaire HAL, est destinée au dépôt et à la diffusion de documents scientifiques de niveau recherche, publiés ou non, émanant des établissements d'enseignement et de recherche français ou étrangers, des laboratoires publics ou privés. 


\title{
Conformal Propagation and Near-Omnidirectional Radiation with Surface Plasmonic Clothing
}

\author{
Xi Tian, Qihang Zeng, Denys Nikolayev, and John S. Ho
}

\begin{abstract}
Wireless technologies are essential components of wearable devices for applications ranging from connected healthcare to human-machine interfaces. Their performance, however, is hindered by the human body, which obstructs the propagation of wireless signals over a wide range of directions. Here, we demonstrate conformal propagation and near-omnidirectional radiation of wireless signals near the body using clothing integrated with spoof surface plasmonic structures. These structures, fabricated entirely from conductive textiles, induce wireless signals emitted by nearby antennas to propagate around the body as surface waves and radiate as propagating waves in directions otherwise obstructed by the body. We describe the procedure for designing textile-based spoof surface plasmon waveguides, radiating elements, and impedance matching sections for operation in the 2.4-2.45 GHz ISM band. Using a tissue phantom model of the human torso, we experimentally demonstrate $\mathbf{2 . 6 2}$ greater angular coverage by a dipole placed near the body compared to without the clothing.
\end{abstract}

Index Terms-Wearable antennas, spoof surface plasmons, smart textiles.

\section{INTRODUCTION}

W IRELESSLY-connected wearable devices have many applications in emerging healthcare, communication, and human-computer interface technologies [1]. To create more functional devices, techniques to increase the performance of the radio-frequency wireless system (for example, $\mathrm{Wi}-\mathrm{Fi}$, Bluetooth, or $4 \mathrm{G} / 5 \mathrm{G}$ ) are essential because it represents a major constraint on the size, power consumption, and lifetime of the device. A major obstacle in the performance of wearable wireless technologies, however, is the presence of the human body. Because the human body obstructs the propagation of radio-frequency waves, the ability of radiofrequency signals to radiate into a wide range of directions around the body is greatly limited. This obstruction results in poor angular coverage that adversely impacts the reliability and energy efficiency of the wearable device.

Antenna design approaches have been extensively investigated to improve the radiation pattern emanating from devices worn on the body [2]-[5]. Classes of antennas developed for use in wearable devices include cavity slot antennas [6], [7],

Manuscript submitted January 26, 2020. This work was supported by the National Research Foundation Singapore (NRFF2017-07) and Ministry of Education Singapore (MOE2016-T2-2-016 and MOE2016-T3-1-004). (Xi Tian and Qihang Zeng contributed equally to this work.) (Corresponding authors: Xi Tian and John S. Ho.)

Xi Tian, Qihang Zeng, and John S. Ho are with the Department of Electrical and Computer Engineering, Institute for Health Innovation and Technology, and N.1 Institute at the National University of Singapore, Singapore, (email: tianxi@u.nus.edu; johnho@nus.edu.sg). Denys Nikolayev is with Univ Rennes, CNRS, Institut d'Electronique et de Télécommunications de Rennes, UMR-6164, FR-35000 Rennes, France

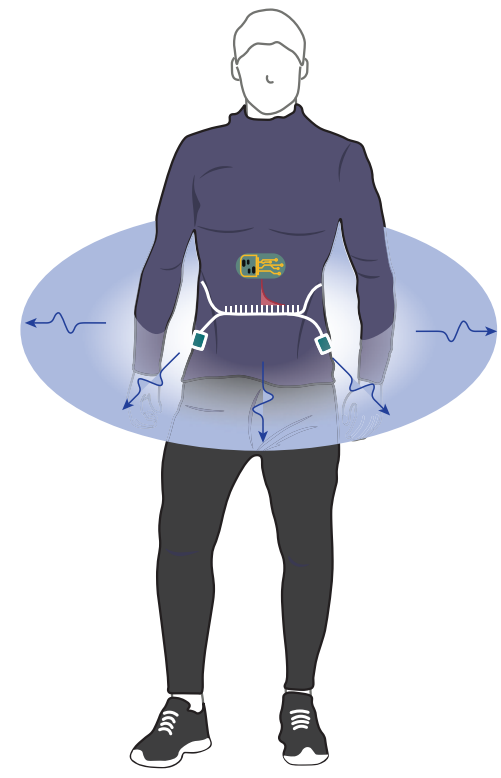

(a)
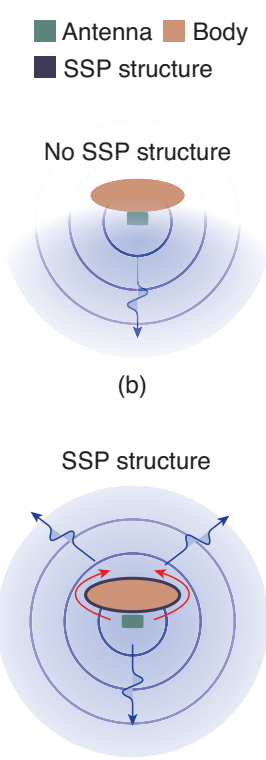

(c)
Fig. 1. Function of the surface plasmonic clothing. (a) Illustration of spoof surface plasmonic (SSP) structure on clothing. (b) Without the structures, the body obstructs the propagation of radio-frequency waves. (c) The structure induces the emitted wireless signals to propagate as surface waves around the body (red arrow) and then radiate around the body (blue arrows).

planar inverted-F antennas [8], [9], crosspatch antennas [10], [11], higher-mode antennas [12], metallic button antennas [13], [14], and steerable-beam antennas [15]-[17]. Although these designs exhibit useful properties both on and off the body, they do not address the obstruction of wave propagation and remain limited in angular coverage when worn on the body. Another approach is to integrate the antennas into clothing, such as by fabricating them using conductive textile materials [18]-[21]. By allowing multiple antennas to be placed on the body [22], this strategy can potentially circumvent obstruction and provide near-omnidirectional coverage around the body. However, current implementations rely on wired connections between the antennas and the signal source. These connectors present a major inconvenience to the user, and reduce the robustness of the clothing to washing and daily wear.

Recent advances in metamaterials have inspired the development of a class of thin, flexible waveguides that support spoof surface plasmons (SSP) at radio-frequencies [23]. These structures have been used to create a wide range of microwave components and devices [24]-[26], including ultrawideband filters [27], [28], ultrathin ring resonators [29], and high- 
isolation power dividers [30]. We have recently integrated such waveguides into clothing using conductive textile materials [31]. Such clothing can significantly increase the efficiency of wireless transmission between wearable devices because of the excitation of surface waves by nearby antennas: wireless signals emitted by devices near the clothing can be induced to propagate conformally around the body rather than to radiate into the surrounding space. Importantly, these designs exploit the evanescent field associated with SSP modes to enable excitation by proximity to existing wireless devices without requiring connectors to the clothing. To interface with external devices, however, the integration of antennas and impedance matching sections is required to launch surface waves into radiating waves in the desired directions. Although their potential for integration with antennas was previously shown, the design of textile structures capable of redirecting radiation from a nearby devices to overcome obstruction by the body has not been previously explored.

In this paper, we demonstrate a surface plasmonic clothing that enables conformal propagation and near-omnidirectional radiation of wireless signals around the body. To overcome the obstruction of radiation by the body [Fig. 1(a)], our design incorporates several advances over prior work: (i) an optimized design procedure for textile-based SSP waveguides and patch antennas for launching surface waves, (ii) the integration of impedance matching sections, and (iii) detailed measurement and analysis of the radiation pattern generated around the body. We analyze the excitation of the surface modes and describe the procedure for designing such structures, including the SSP waveguide, radiating elements, and impedance matching section, for operation in the $2.4-2.5 \mathrm{GHz}$ industrial, scientific, and medical (ISM) band. We fabricate the clothing using thin conductive textiles attached to a fabric substrate and experimentally demonstrate near-omnidirectional radiation around a tissue phantom with angular coverage 2.62 times greater than without the clothing.

\section{CONCEPT AND DESIGN}

\section{A. Surface Plasmonic Clothing Concept}

Fig. 2 illustrates the design of the textile structure comprising of a SSP waveguide, matching sections, and radiating elements. When a radiating antenna is placed in close proximity to the SSP waveguide, the interaction between the source current distribution and the evanescent field of the surface mode induces a portion of the outgoing energy to propagate as surface waves. These surface waves propagate conformally along the waveguide, following the curvature of the body without reflection. Impedance matching sections then enable the surface waves to excite radiating elements placed around the body. These radiating elements launch the surface waves as radiation into the surrounding space, thereby enabling the radiation pattern emanating from the body to cover directions otherwise obstructed by the body. Crucially, the surface plasmonic structure affords control over the propagation and radiation of wireless signals through passive proximity to wirelessly-enabled wearable devices. The clothing enables the field radiated by a wearable device to be redirected around

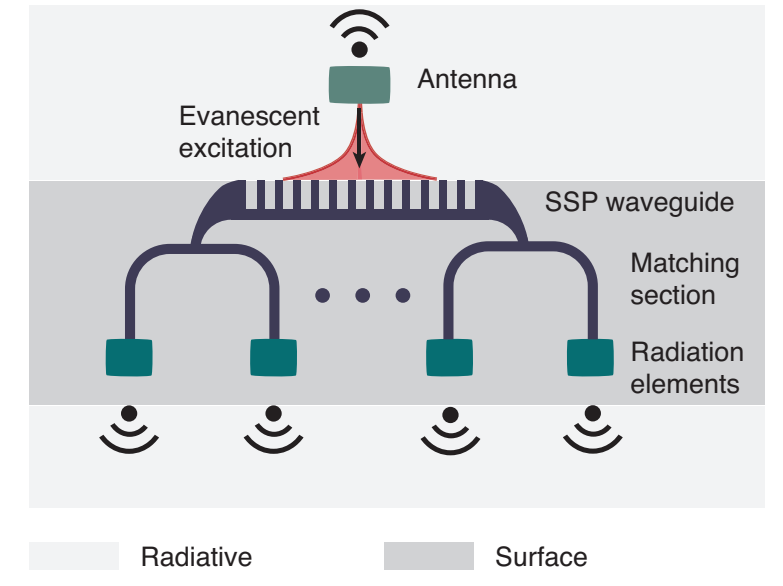

Fig. 2. Surface plasmonic clothing design. Antennas placed in proximity to the SSP waveguide excite surface waves, which are guided to radiating elements that launch propagating waves. Matching sections connect the surface waves to the radiating elements.

the body without physical connectors or modification of its built-in antenna.

Several design considerations are essential to implement the surface plasmonic clothing. First, the SSP waveguide must support surface plasmon-like modes over the operating frequency range. Second, the modes should be tailored to enable contactless excitation using antennas placed in close proximity to the body. Third, the transmission line needs to be interfaced to radiating elements that efficiently convert the surface wave into a propagating wave. Finally, the SSP structure must be fabricated using conductive textiles and integrated into clothing.

\section{B. Surface Plasmonic Waveguides}

We describe the design of the textile-based SSP waveguide in order to support surface modes with the desired properties over the operating frequency band. The design process uses a simplified model of the body comprising of a half-space in which the upper region $(z>0)$ is filled with freespace and a lower region $(z<0)$ with biological tissue of dielectric permittivity $\varepsilon_{\text {body }}$. The textile structure is placed on the interface $z=0$ and supports surface-plasmon-like modes propagating in the $\pm x$ directions.

We first introduce the design parameters by providing an approximate description of the surface modes. While the modes exhibit complex field distributions that depend on the detailed textile structure, the electric field can in general be approximated by the form

$$
\mathbf{E}_{ \pm}(\mathbf{r})=p(y)\left(\begin{array}{c}
i \alpha_{n} / \beta \\
0 \\
1
\end{array}\right) e^{ \pm i \beta x-\alpha_{n}|z|}
$$

where $p(y)$ describes the structure-dependent field profile in the $y$ direction, $\alpha_{n}$ are the decay constant in the upper $(n=1)$ and lower $(n=2)$ regions, $\beta$ is the propagation constant, and a time-dependency of $e^{-i \omega t}$ has been assumed [32]. In addition to the field distribution described in Eq. (1), the structure should also support surface-plasmon-like dispersion

\section{ACCEPTED MANUSCRIPT}




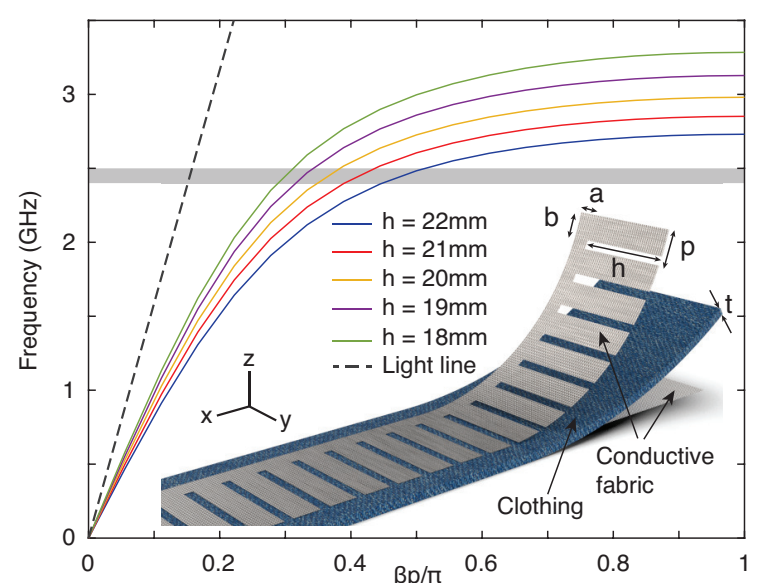

(a)
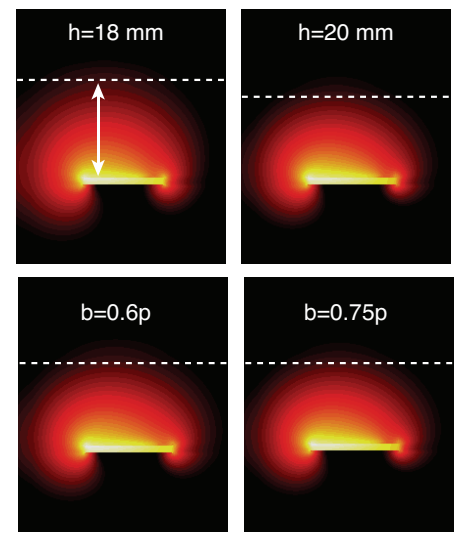

0

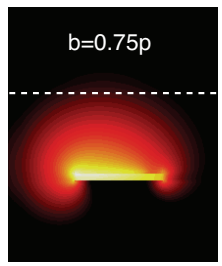

$\log \left|E_{z}\right|(a . u$.

(c)

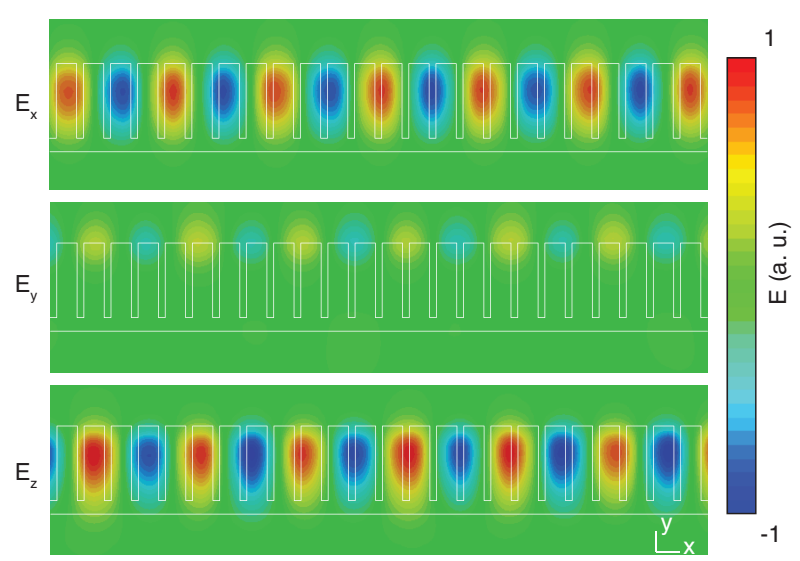

(b)
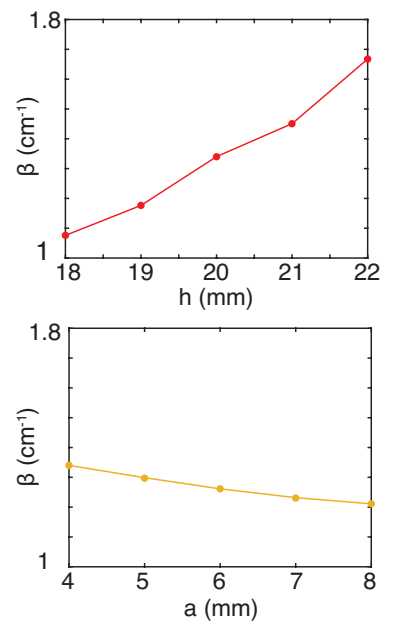

(d)
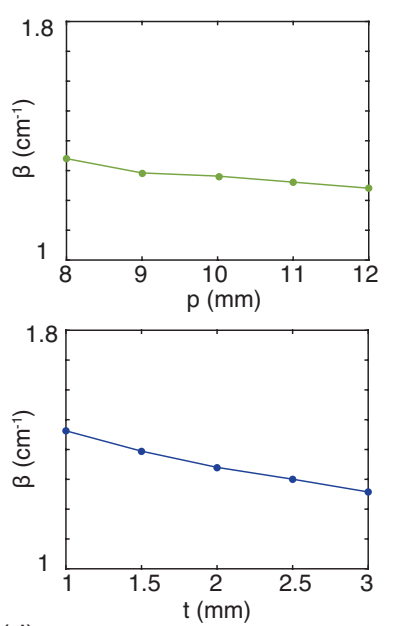

d)

Fig. 3. Textile SSP waveguide design. (a) Geometrical parameters of the structure and dispersion curves for varying $h$. The remaining parameters are $a=4 \mathrm{~mm}, b=6 \mathrm{~mm}, p=8 \mathrm{~mm}, t=2 \mathrm{~mm}$, and $\varepsilon_{\text {fabric }}=1.5$ ). (b) Electric field of the mode in the $x y$ plane at $2.45 \mathrm{GHz}$ for $h=21 \mathrm{~mm}$. (c) Electric field of the mode in the $y z$ plane for varying $h$ and $b$. White dashed lines show the decay length $3 \alpha^{-1}$. (d) Propagation constant $\beta$ as a function of $h, p, a$, and $t$.

characteristics. In the simplest approximation, the propagation constant $\beta$ follows the surface plasmon dispersion relation $\beta=k_{0} \sqrt{\varepsilon_{1} \varepsilon_{2} /\left(\varepsilon_{1}+\varepsilon_{2}\right)}$, where $k_{0}=\omega / c$ is the wavenumber and $\varepsilon_{2}=1-\omega_{p}^{2} / \omega^{2}$, with both $\varepsilon_{1}$ and $\omega_{p}$ as fitting parameters. This relation results in a dispersion curve that lies to the right of the light line $\beta=k_{0}$ and approaches a horizontal asymptote at the frequency $\omega_{s p}=\omega_{p} / \sqrt{1+\varepsilon_{1}}$. The field distribution and dispersion relation together imply a bound surface mode that is analogous to an optical surface plasmon polariton at the metal-dielectric interface, although the material parameters $\varepsilon_{1}$ and $\varepsilon_{2}$ should be strictly regarded as fitting parameters without their usual physical interpretation.

The structure of the SSP textile consists of a planer patterned top layer, an intermediate fabric layer, and an unpatterned bottom layer as shown in Fig. 3(a). In the upper region $z>0$, the mode has a decay constant $\alpha_{1}$ given by $\alpha_{1}=\sqrt{\beta^{2}-k_{0}^{2}}$. Since the dispersion relation requires $\beta>k_{0}, \alpha_{1}$ is purely real and the mode does not radiate into the upper region $z>0$. The addition of the unpatterned bottom layer prevents the evanescent field from extending into the lower region $\alpha_{2} \rightarrow \infty$, which introduces additional radiative losses into the body region due to coupling to modes with propagation constant $\beta<\omega \sqrt{\varepsilon_{\text {body }}} / c$. While analytical expressions for dispersion relation supported by the SSP waveguides in unbounded free space are available [33], the increased complexity of our structure requires numerical simulation to obtain the surface mode characteristics.

To design the SSP structure, we use an eigenmode solver of CST Microwave Studio (Dassault Systems). Unit-cell analysis of the structure yielded the dispersion curves on Fig. 3(a) corresponding to a set of geometrical parameters. These dispersion curves exhibit the surface-plasmon-like characteristics with properties that can be tuned by varying the geometry. Using this approach, the geometrical parameters can be optimized to achieve a desired $\beta$ and mode field distribution at a particular operating frequency.

Fig. 3(b)-(c) show the electric field of the SSP mode for different geometrical parameters. The field is strongly confined within the width of the structure, but extends evanescently above the surface. The decay length $\alpha_{1}^{-1}$ is primarily controlled by the comb height $h$ [23], and is largely insensitive to the other comb parameters over a wide range of values 
[Fig. 3(d)]. The region between the top comb structure and bottom unpatterned layer is characterized by the thickness $t$ and permittivity $\varepsilon_{\text {fabric }}$ of the underlying fabric, which are generally selected based on wearability considerations. The SSP mode exhibits a weak dependence on these parameters, which can be compensated by tuning $h$ to obtain the required $\beta$. Using cotton fabric $\left(\varepsilon_{\text {fabric }}=1.5\right)$ with thickness $t=2 \mathrm{~mm}$, we obtained the structure parameters $a=4 \mathrm{~mm}, b=6 \mathrm{~mm}$, $h=21 \mathrm{~mm}$, and $p=8 \mathrm{~mm}$ to achieve $\beta=0.46 \pi \mathrm{cm}^{-1}$ at $2.4 \mathrm{GHz}$. The dimensions of the structure represent feature sizes that are well within the tolerances of textile cutting processes.

\section{Surface Mode Excitation}

Owing to the evanescent field extending from the surface of the SSP textile, the surface modes can be wirelessly excited by nearby antennas without physical connectors. In contrast, most conventional microwave waveguides and transmission lines strongly confine the modal field within its structure and require connectors to efficiently interface with external devices. The surface excitation effect can be described by separating the electric field of the surface mode into forward $(+)$ and backward (-) propagating components

$$
\mathbf{E}(\mathbf{r})=a_{+} \mathbf{E}_{+}(\mathbf{r})+a_{-} \mathbf{E}_{-}(\mathbf{r}),
$$

where the $a_{ \pm}$is the mode amplitude and $\mathbf{E}_{ \pm}$are normalized such that $\frac{1}{2} \int|p(y)|^{2} d y=\alpha_{1} \alpha_{2} /\left(\alpha_{1}+\alpha_{2}\right)$. When a source with current density $\mathbf{J}(\mathbf{r})$ is placed above the SSP clothing, the surface modes are excited with amplitude [34, Ch. 8.12.C]

$$
a_{ \pm}=-\frac{\beta}{2 \omega \varepsilon_{0}} \int \mathbf{J}(\mathbf{r}) \cdot \mathbf{E}_{\mp}(\mathbf{r}) d \mathbf{r} .
$$

Equation (3) shows that excitation depends only on the overlap between the current density and the evanescent field above the surface. The current distribution can be provided by a radiating antenna placed in close proximity to the structure, in which case the excitation of surface modes is accompanied by suppression of radiation into the surrounding space. As the excitation occurs in the near-field of the antenna, far-field properties such as gain and directivity do not play a direct role in determining the amplitude of the excited mode.

We characterize the efficiency of surface mode excitation at $2.4 \mathrm{GHz}$ using a dipole antenna of resonant length. The excitation efficiency is defined as $\eta=P_{\text {ssp }} / P_{\text {in }}$, where $P_{\text {in }}$ is the input power into the dipole antenna and $P_{\text {ssp }}$ is the power leaving ports in the $\pm x$ directions of the SSP waveguide. Since the surface plasmon modes are transverse-magnetic, the transverse $y$ components of the electric field is negligible [Fig. 3(b)] and the surface modes are predominantly excited when the dipole is oriented in the $x$ or $z$ directions. Fig. 4(a) shows the theoretically calculated $\eta$ as a function of the distance $d$ above the surface for a $x$-oriented dipole, using (3) and analytical expressions for the electric field and dipole current distribution. The excitation efficiency is high when $d$ is comparable to the decay length $\alpha_{1}^{-1} \approx 7 \mathrm{~mm}$ above the surface. As $d$ decreases, excitation of the surface wave simultaneously suppresses radiation from the dipole, as shown

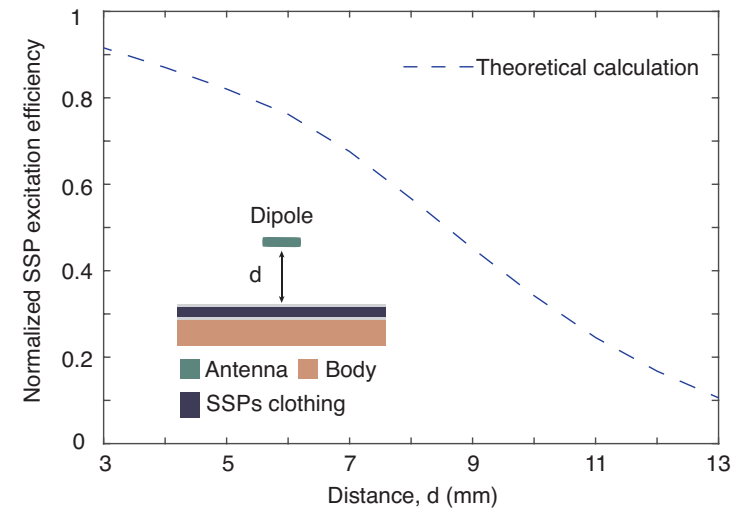

(a)

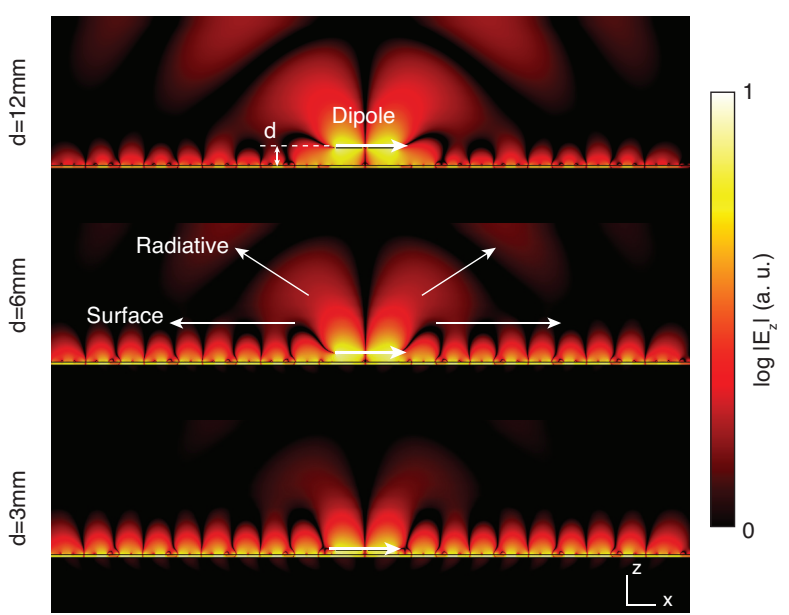

(b)

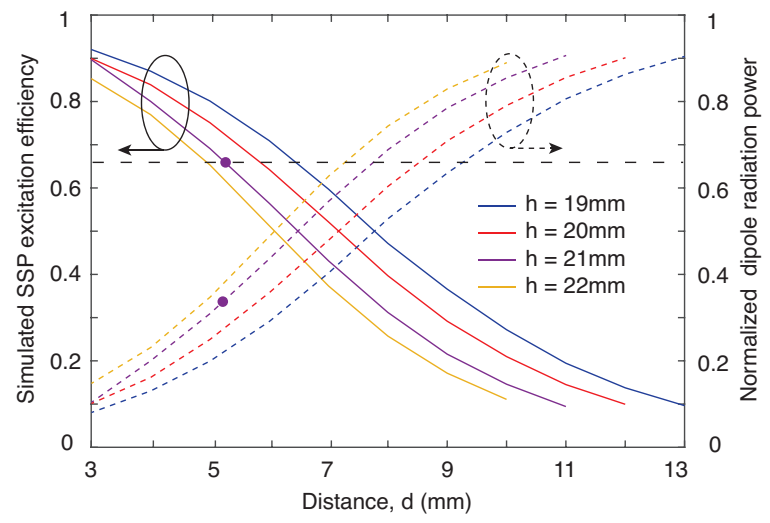

(c)

Fig. 4. Excitation of surface modes. (a) Theoretically calculated excitation efficiency of the surface modes for $h=19 \mathrm{~mm}$. (b) Electric field profile when an $x$-oriented electric dipole $(2.4 \mathrm{GHz}$ resonant length) is placed at distance $d$ above the surface. (c) Simulated excitation efficiency of surface modes as a function of $d$ for varying waveguide width $h$.

in the full-wave simulations in Fig. 4(b). This model yields an excitation efficiency in close agreement with analytical calculations, and shows that the power input into the antenna is transferred from radiation to surface modes [Fig. 4(c)].

To generate near-omnidirectional radiation, the excitation distance $d$ should be fixed to provide the surface excitation necessary to radiate power equally in each direction. For the case of two secondary radiating elements—each excited by the

\section{ACCEPTED MANUSCRIPT}


surface wave propagating in the $\pm x$ direction-the excitation efficiency required is $\eta=2 / 3$ such that the remaining $1 / 3$ energy is directly radiated by the dipole. The designed SSP structure with $h=21 \mathrm{~mm}$, as shown in Fig. 3(a), achieves this target efficiency when $d$ is approximately $5 \mathrm{~mm}$. In practice, $d$ is largely determined by the design of the wearable device, such as thickness of the packaging around the antenna, and the manner by which it is worn on the body. The optimal $d$ can be tuned over a wide range by varying the parameter $h$ to control the decay constant $\alpha$ using the previously described procedure.

\section{Radiating Elements and Matching Section}

Conversion of surface waves into radiation is required to provide coverage in desired directions around the body. Previous studies of surface-to-propagation wave conversion for the SSP structure provide the basis for the design of such elements [35], [36], although the structure must satisfy additional requirements in order to be integrated into clothing and achieve near-omnidirectional radiation. First, radiation from each element should be single-sided to prevent transmission into the human body, which results in losses and tissue heating. Second, the structure should be simple and planar to be compatible with textile fabrication processes and allow easy integration into clothing. Third, the directivity should be sufficient to provide the required angular coverage in each direction without interfering with adjacent elements.

To meet these criteria, we use microstrip patch antennas as the radiating elements due to their ease of fabrication, single-sided radiation characteristics, and low directivity. The interface between the SSP waveguide and the patch antenna uses an impedance matching section, while the ground plane is simply extended over the length of the patch antenna [Fig. 5(a)]. We design the impedance matching section using a standard gradient corrugated strip with a linear taper by varying the length of the matching section and identifying the minimum reflection to a port $50 \Omega$ [27], [37]-[39]. The two conductive fabric layers are modeled using with a thickness of $0.1 \mathrm{~mm}$ and separated by a $2 \mathrm{~mm}$ dielectric substrate with $\varepsilon_{\text {fabric }}=1.5$ and loss tangent $\tan \delta=0.04$ [40] to model the textile substrate.

Fig. 5(b) shows the simulated electric field in the $x z$ plane cutting through the center of the patch antenna when the structure is placed on a planar interface between air and tissue $\left(\varepsilon_{r}=50\right)$. The device efficiently converts surface waves excited by energy input into from a waveguide port into radiation into the upper half-space with minimal reflection $\left|S_{11}\right|<10 \mathrm{~dB}$ over the 2.4-2.45 GHz band [Fig. 5(c)]. Due to the ground plane, the presence of the body does not significantly affect the performance of the structure.

\section{E. Integration into Clothing}

To demonstrate control of radiation around the body, we design the textile structure shown in Fig. 6(a) for integration into clothing. The structure consists of a SSP waveguide with 35 unit cells that terminates at both ends with a matching section and a secondary radiating element. The entire structure
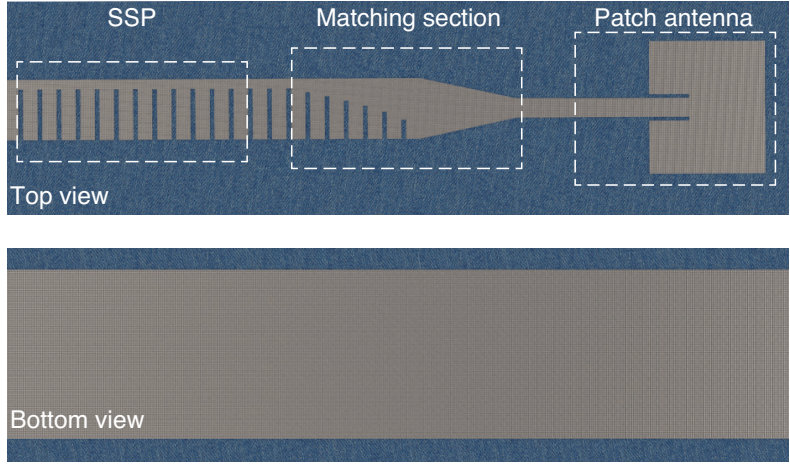

(a)

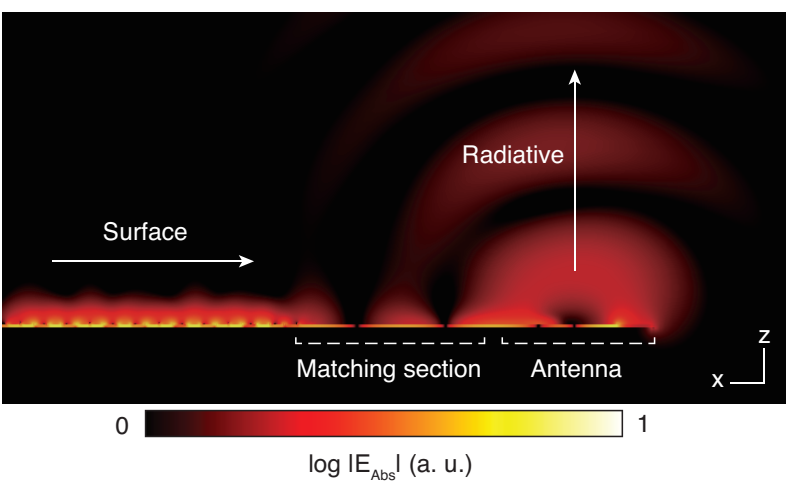

(b)

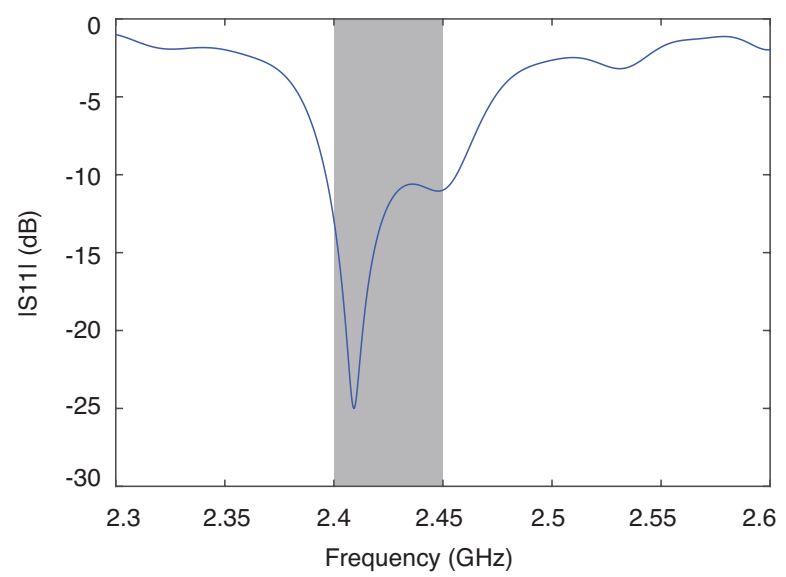

(c)

Fig. 5. Radiative elements and matching section. (a) Schematic of structure including the SSP waveguide, matching section, and antenna. (b) Electric field magnitude in the $x z$ longitudinal plane for excitation of the SSP waveguide. (c) Simulated $\left|S_{11}\right|$ of the radiating element.

wraps partially around the waist with a separation angle of $\theta$, defined from the center of each radiating element, as illustrated in Fig. 6(b)-(c). When a radiating antenna is placed near the front of the body, energy is directed to propagate as surface waves around the body and radiate into backwards direction. As a result, the resulting radiation pattern can be nearly omnidirectional, even when the antenna is obstructed by the body. Although we use two secondary radiating elements to demonstrate this effect, the structure can be extended to incorporate additional radiating elements, each with higher

\section{ACCEPTED MANUSCRIPT}



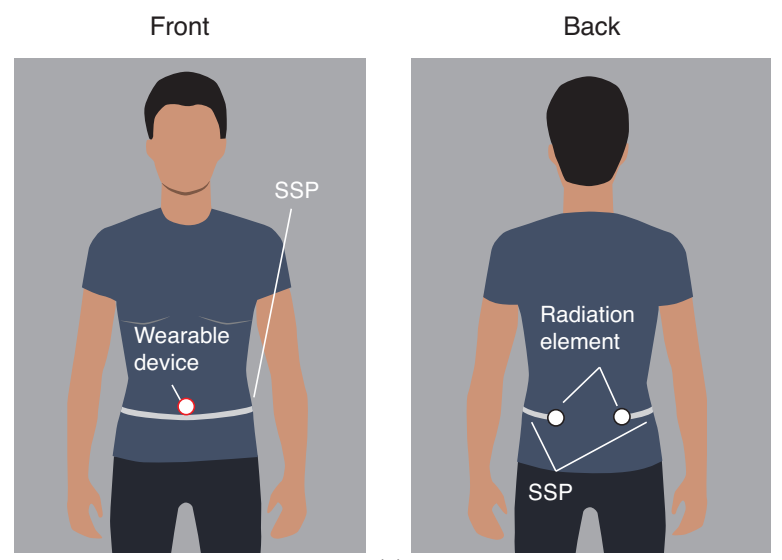

(a)

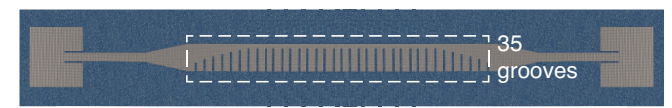

(b)

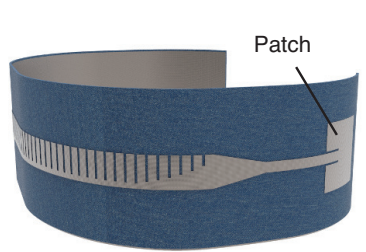

(c)

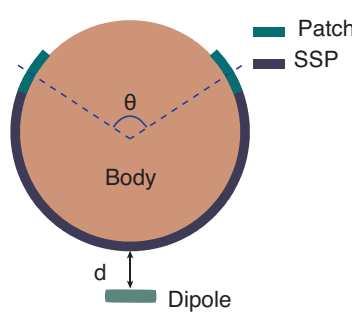

(d)
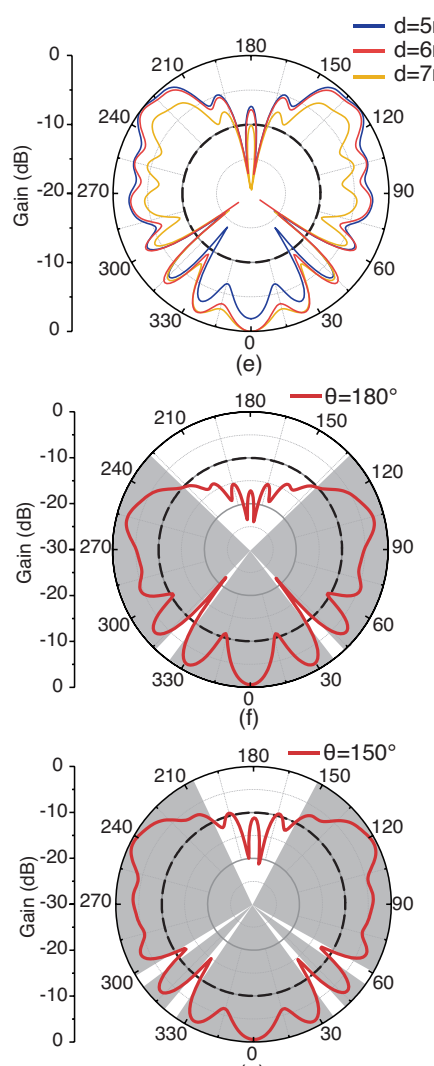

0
$(\mathrm{~g})$

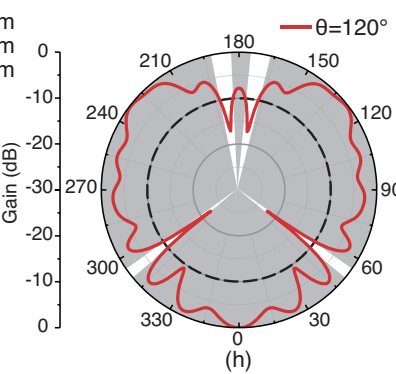

(h)
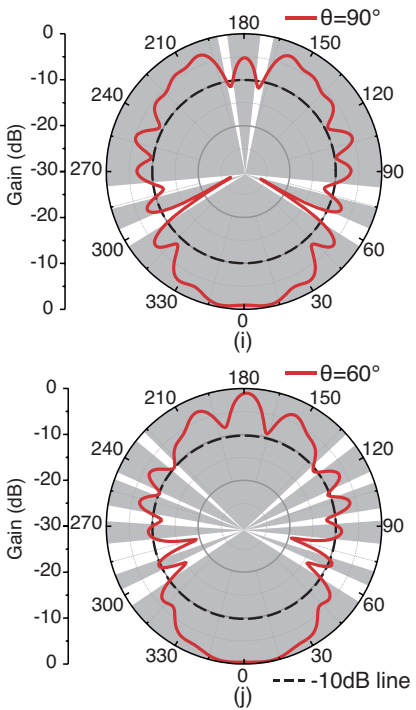

Fig. 6. Surface plasmonic clothing design. (a) Illustration of clothing integrated with the SSP waveguide and two secondary radiating elements for redirecting radiation from a device worn on the front of the body. (b) Top view of the flattened structure. (c) Side view of the curved structure. (d) Cross-section view of the structure on a simplified cylindrical model of the body. The angular separation between the radiating elements is $\theta$ and $d$ is the distance of the dipole antenna (representing the wearable device) $d$ from the surface. (e) Simulated radiation patterns for $d=5-7$ mm. (f)-(j) Simulated radiation patterns for $\theta=60-180^{\circ}$. Shaded area indicates the angular coverage where the gain is higher than $-10 \mathrm{~dB}$.

directivity, in order to achieve greater control over the radiation pattern.

We characterize the radiation pattern generated when the structure wrapped around a cylindrical model of the human torso $\left(40-\mathrm{cm}\right.$ diameter, $\left.\varepsilon_{\text {body }}=40\right)$ and a dipole antenna placed at distance $d$ above surface. The radiation patterns in Fig. 6(e) show the angular coverage for $d=5-7 \mathrm{~mm}$. The angular coverage is maximized when $d=6 \mathrm{~mm}$, which is in close agreement with the distance corresponding to the required excitation efficiency in Fig. 4(c). The separation angle $\theta$ between the radiating elements affects the angular coverage as well. Compared with the $-3 \mathrm{~dB}$ threshold widely used to measure the directivity of antennas in free-space, here we use a lower reference threshold of $-10 \mathrm{~dB}$ in order to quantify the angular coverage around the body relative to the radiation pattern of a dipole placed in front of the body, which has a front-back ratio that exceeds $20 \mathrm{~dB}$. As shown in Fig. $6(\mathrm{~g})-(\mathrm{j})$, the radiation pattern is nearly omnidirectional for $\theta=60^{\circ}-150^{\circ}$. The angular coverage is maximized at $\theta=120^{\circ}$ when the radiating elements and dipole antenna are equally distributed around the body.

Fig. 7 compares the absolute gain of each antenna for $\theta=120^{\circ}$. These results demonstrate two key reasons why SSP clothing could improve the angular coverage. First, the angular redistribution of the radiated energy mitigates the body obstruction. Second, similar to the dipole with a ground plane in Fig. 7(b), the minimization of power absorption in tissues increases the total energy radiated into the surrounding space. For this reason, the SSP clothing enhances the angular coverage with minimal penalty on the broadside gain $(<5 \mathrm{~dB}$, compared with dipole on the body without a ground plane).

We perform a full-wave simulation of the structure using a dipole antenna is placed on the center of the abdomen $6 \mathrm{~mm}$ above the transmission line. The stimulations use a voxel model of the human body (Donna, CST Microwave Studio), which captures the tissue heterogeneity of the body. Fig. 8(a)(b) show the simulated electric field profile for the dipole antenna without and with the SSP device. A significant portion of the energy leaving the antenna can be seen to propagate as surface waves around the body, which radiate in the backward directions. As demonstrated in previous studies [23], propagation of the SSP waveguide is highly robust to bending and deformation. Along the curvature of the body, simulations show that reflection losses are about $3.9 \%$ compared to the flat structure and propagation losses about $1.5 \%$. Fig 8(c)(d) show the corresponding $x y$-plane radiation patterns at $2.4 \mathrm{GHz}$ and $2.45 \mathrm{GHz}$. Due to obstruction by the body, the angular coverage provided by the dipole in absence of the SSP

\section{ACCEPTED MANUSCRIPT}



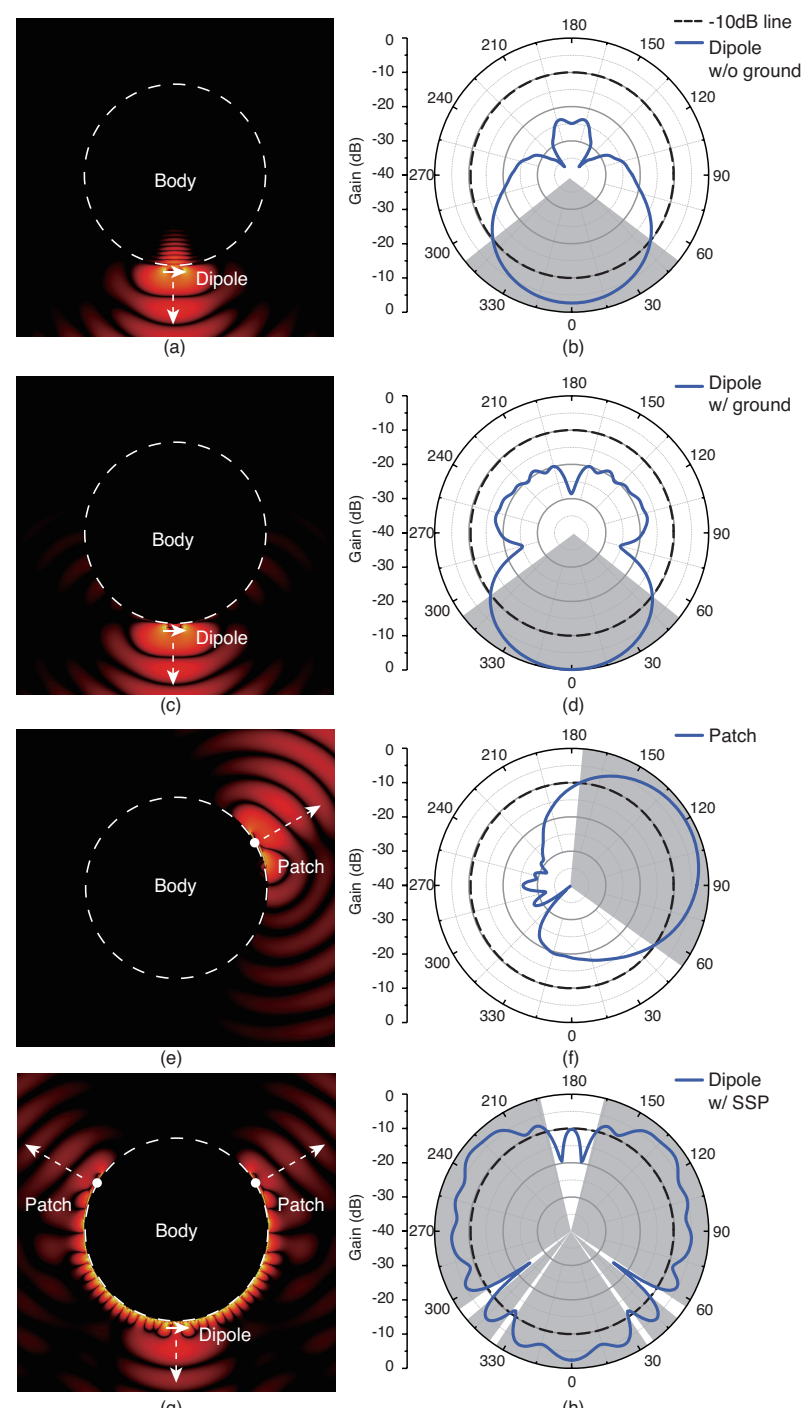

(g)

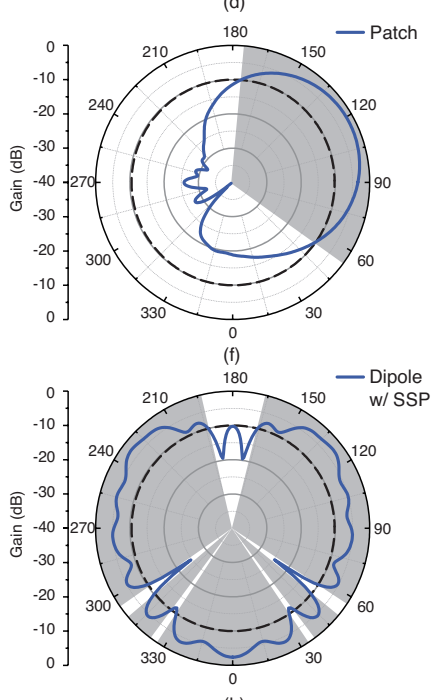

(h)
Fig. 7. Antenna radiation and gain on a cylindrical model of the human torso. (a)-(b) Dipole without ground, (c)-(d) dipole with ground, (e)-(f) textilebased patch antenna, and $(\mathrm{g})-(\mathrm{h})$ dipole with the SSP textile structure. The gain is normalized to the overall highest gain, which is $4.19 \mathrm{~dB}$ for the dipole with ground. The cylinder has a diameter of $40 \mathrm{~cm}$ and a uniform dielectric permittivity of $\varepsilon_{\text {body }}=40$.

clothing is $114^{\circ}$ at $2.4 \mathrm{GHz}$, compared to $278^{\circ}$ in free-space. When placed near the SSP clothing, however, the angular coverage is increased about 2.7 times to $314^{\circ}$, providing nearly omnidirectional radiation. The simulated angular coverage results are summarized in Table I.

\section{EXPERIMENTAL DEMONSTRATION}

\section{A. Experimental Setup}

We experimentally demonstrate the operation of the clothing by measuring the radiation pattern emanating from a $20 \mathrm{~cm}-$ diameter cylindrical tissue phantom to mimic the human body. The surface plasmonic clothing is fabricated by using a laser cutter to pattern the structure from thin conductive textiles $\mathrm{Cu} / \mathrm{Ni}$ nylon ripstop fabric sheets (Conductive Fabric 4713, Holland Shielding Systems). The textiles are then attached to

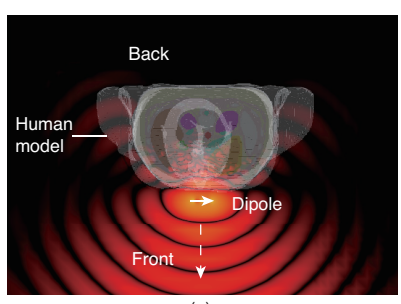

(a)
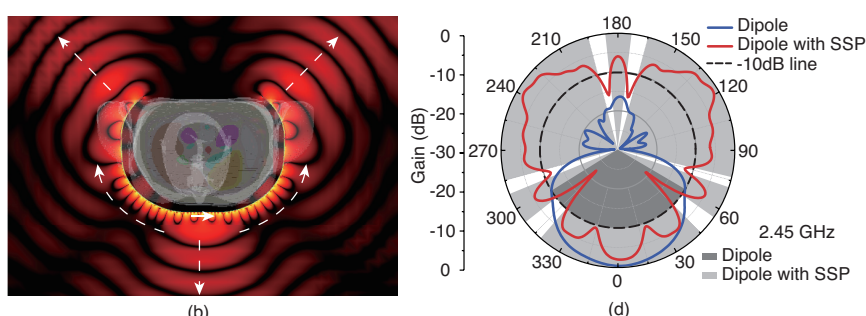

Fig. 8. Antenna radiation in a computational human body model. (a)-(b) Simulated electric field in the transverse plane of the body (a) without and (b) with the SSP clothing when a dipole antenna (solid white arrow) is placed near the front of the body. Dashed white lines indicate the direction of wave propagation. (c)-(d) Radiation patterns with and without the surface plasmonic clothing at (c) $2.4 \mathrm{GHz}$ and (d) $2.45 \mathrm{GHz}$. The shaded areas indicate the angular coverage where the gain is greater than $-10 \mathrm{~dB}$.

a cotton fabric sheet using a fabric adhesive. The resulting device is free of connectors and is entirely made of textile, which enables it to conform to the body when worn and be robust to washing. To test the clothing, the device is wrapped conformally around the cylinder and excited using a wideband dipole antenna resonant at $2.4 \mathrm{GHz}$ placed at a distance $d$ above the surface. The entire setup is placed on a rotating stage in an anechoic chamber as shown in Fig. 9(a).

The tissue phantom comprises of a cylindrical polypropylene container (20-cm diameter) filled with an agarose gel to mimic the shape and electromagnetic properties human torso. The phantom solution uses agarose, water and sugar following the recipe described in Ref. [41]. We verified the phantom solution by measuring the dielectric permittivity spectrum with an open-ended dielectric probe. As shown in Fig. 9(b), the dielectric permittivity is approximately $\varepsilon_{r}=54$ within the 2.4-2.5 GHz ISM band, which is close to permittivity of muscle tissue.

TABLE I

Simulated Angular Coverage in Computational Model of the HUMAN BODY

\begin{tabular}{lcccc}
\hline \hline \multicolumn{5}{c}{ Angular coverage (Gain $>-10 \mathrm{~dB})$} \\
\hline Frequency & $\begin{array}{c}\text { Dipole } \\
\text { (free space) }\end{array}$ & $\begin{array}{c}\text { Dipole } \\
\text { (w/o ground) }\end{array}$ & $\begin{array}{c}\text { Dipole } \\
\text { (w/ ground) }\end{array}$ & $\begin{array}{c}\text { Dipole } \\
\text { (w/ SSP) }\end{array}$ \\
\hline $2.4 \mathrm{GHz}$ & $278^{\circ}$ & $114^{\circ}$ & $112^{\circ}$ & $314^{\circ}$ \\
\hline $2.45 \mathrm{GHz}$ & $277^{\circ}$ & $116^{\circ}$ & $111^{\circ}$ & $304^{\circ}$ \\
\hline \hline
\end{tabular}




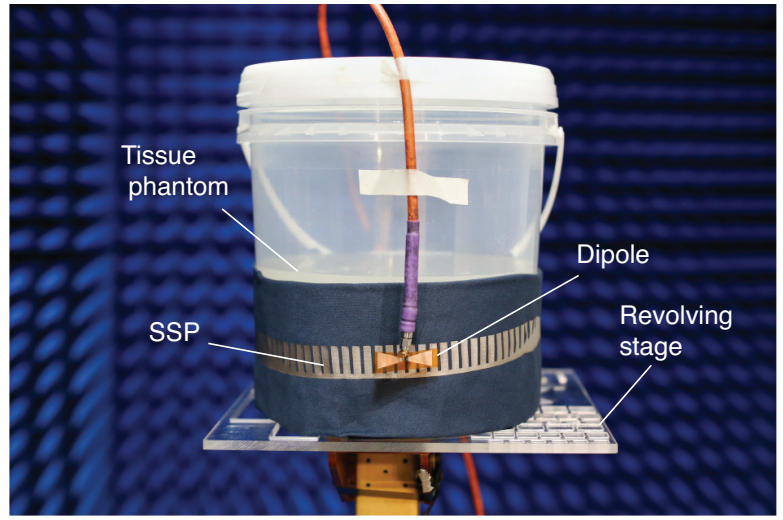

(a)

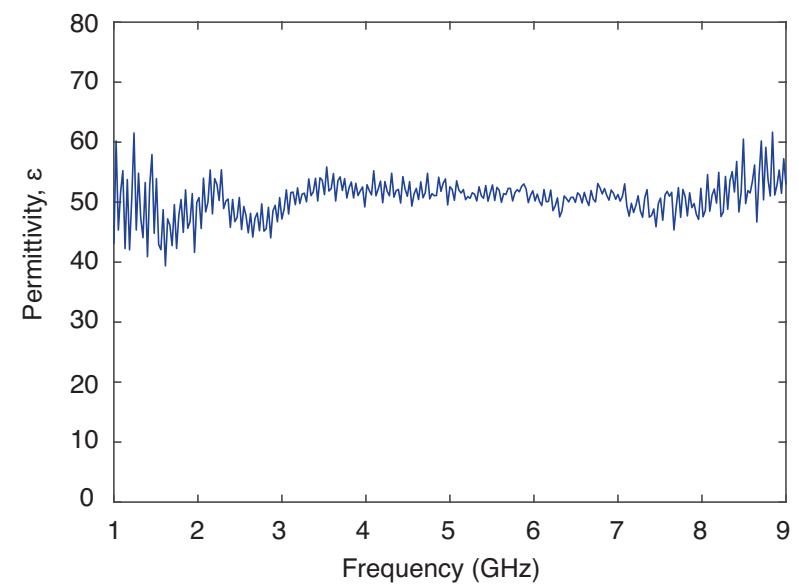

(b)

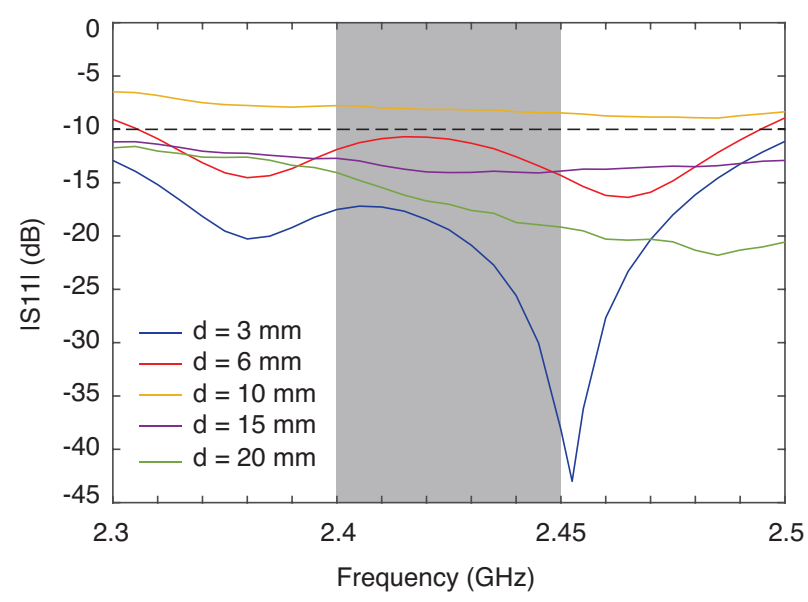

(c)

Fig. 9. Experimental setup. (a) Measurement setup using a 20-cm diameter cylindrical container filled with tissue-mimicking hydrogel. (b) Measured dielectric permittivity of hydrogel as a function of frequency. (c) Measured $\left|S_{11}\right|$ at different excitation distance $d$.

\section{B. S-parameter Measurements}

We measured the scattering parameter $S_{11}$ to characterize the effect of proximity to the SSP structure on the power leaving the dipole. Fig. 9(c) shows the $\left|S_{11}\right|$ spectrum for $d$ varying from $3 \mathrm{~mm}$ to $20 \mathrm{~mm}$. At close proximity (up to $6 \mathrm{~mm})$, the $\left|S_{11}\right|$ curve exhibits two dips located at about
2.36 GHz and $2.45 \mathrm{GHz}$, and is lower than $-10 \mathrm{~dB}$ across the ISM band. The dips correspond to the resonances of the patch antenna as well as the modes associated with the finite length of the SSP waveguide. At about $d=10 \mathrm{~mm}$, the spectral dips vanish, indicating that the SSP mode is weakly excited. Beyond $d=15 \mathrm{~mm}$, the $S_{11}$ spectrum is insensitive to $d$, which is consistent with the dipole directly radiating into the surrounding space without exciting surface modes.

\section{Radiation Pattern Measurements}

We measured the radiation patterns at $2.4 \mathrm{GHz}$ and 2.45 $\mathrm{GHz}$ with and without the SSP clothing on the tissue phantom. Fig. 10(a)-(b) shows the gain normalized to the maximum in the $x y$-plane. In absence of the SSP clothing, the angular coverage at $2.4 \mathrm{GHz}$ is $123^{\circ}$ and follows the typical broadside pattern associated with obstruction of propagation by the body. When the SSP clothing is placed on the phantom, however, energy is radiated in directions obstructed by the tissue phantom, resulting in a nearly omnidirectional angular coverage of up to $322^{\circ}$. The measured radiation patterns exhibit a slight asymmetry due to imperfect alignment between the dipole antenna and body surface, as well as imprecision in the placement of the flexible fabric material against the tissue phantom. However, the measured angular coverage (Table II) is in close agreement with simulations based on the computational human body model (Table I). Notably, the near-omnidirectional pattern is achieved solely by proximity to the SSP clothing, without altering the transmit power or the antenna.

\section{Robustness Analysis}

To study the effect of the surrounding environment on the textile structure, we evaluated SSP waveguide transmission when a human arm, modeled by a cylinder with a diameter of $5 \mathrm{~cm}$ with a uniform dielectric permittivity of $\varepsilon_{\text {body }}=40$, is placed over the structure. The arm represents a "worst case" obstruction since most other common environmental objects have dielectric permittivities that are less than or comparable to fabric. Fig. 11(a)-(b) illustrate the placement of the arm in close proximity to different parts of the SSP clothing. The change in transmission $\Delta\left|S_{21}\right|$ (relative to $\left|S_{21}\right|$ in absence of the arm) becomes significant when the distance of the arm over the SSP waveguide section of the structure $d_{1}$ is less than $1 \mathrm{~cm}$, and exceeds $-3 \mathrm{~dB}$ when $d_{1}<4 \mathrm{~mm}$. However, the

TABLE II

Measured Angular Coverage in Phantom Model of the Human BODY

\begin{tabular}{lcc}
\hline \hline & Angular coverage (Gain $>-10 \mathrm{~dB})$ \\
\hline Frequency & Dipole & With SSP clothing \\
\hline $2.4 \mathrm{GHz}$ & $123^{\circ}$ & $322^{\circ}$ \\
\hline $2.45 \mathrm{GHz}$ & $120^{\circ}$ & $311^{\circ}$ \\
\hline \hline
\end{tabular}




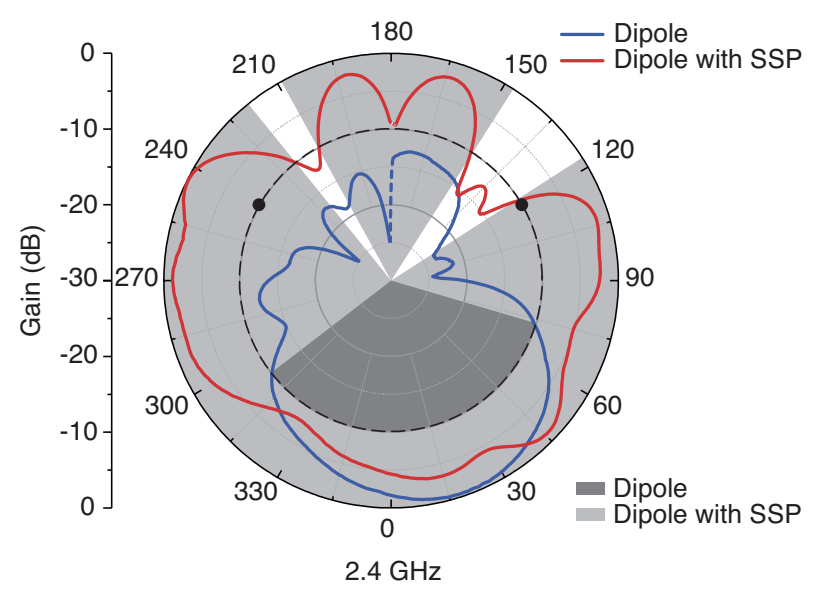

(a)

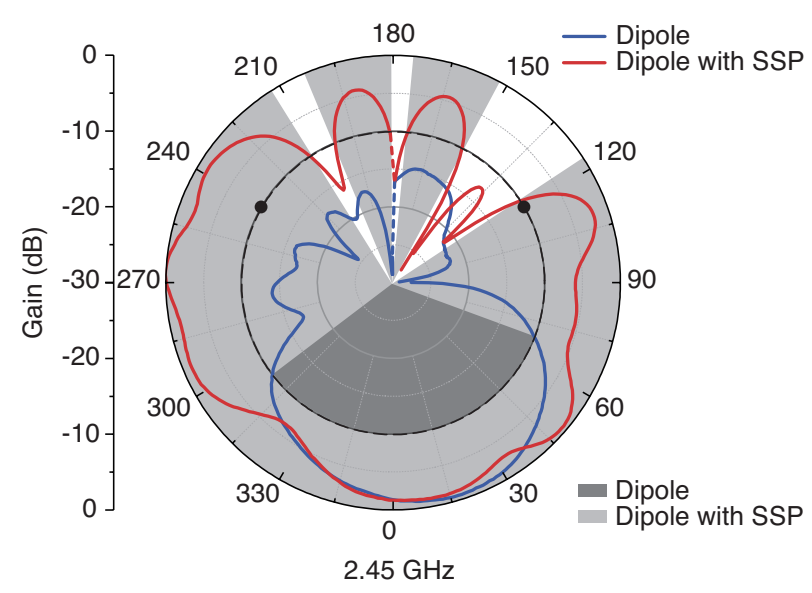

(b)

Fig. 10. Measured radiation patterns with and without the SSP clothing. Radiation pattern measured at (a) $2.4 \mathrm{GHz}$ and (b) $2.45 \mathrm{GHz}$. Shaded areas indicate the angular coverage where the gain is higher than $-10 \mathrm{~dB}$. Black circles indicate angular position of the secondary radiating elements.

drop in $\left|S_{21}\right|$ remains less than the enhancement in front-back ratio of the radiation pattern around the body provided by the SSP clothing $(>10 \mathrm{~dB})$ even when $d_{1}=2 \mathrm{~mm}$. The matching section and microstrip line are relatively insensitive to the presence of the arm with $\Delta\left|S_{21}\right| \approx 1 \mathrm{~dB}$ when $d_{1}=2 \mathrm{~mm}$. These results suggest that careful placement of the matching sections at positions where the human arm usually locate, as is done in our design, can mitigate performance variations due to posture while allowing interactions with nearby antennas over a wide range of positions.

Fig. 11(c)-(d) show another effective approach to reduce the sensitivity of the SSP waveguide by adding an additional fabric layer over sections of the SSP waveguide where contact is expected. The additional fabric layer significantly reduces the effect of the arm, and can enable effective transmission $\Delta\left|S_{21}\right|<4 \mathrm{~dB}$, which maintains significant enhancement in radiation in the backward direction, even at contact $d_{2}=0 \mathrm{~mm}$ for a superstrate thickness of $t=4 \mathrm{~mm}$.

In addition to its use for connector-free interfacing with nearby antennas, the SSP waveguide can also provide distinct advantages in robustness to mechanical damage. Fig. 11(e) illustrates a SSP waveguide and a microstrip line cut with a $0.5-\mathrm{mm}$ gap to mimic mechanical tear during wearing. Simulations of the time-averaged electric field show that the surface wave propagating along the SSP waveguide can significantly traverse the gap, whereas the microstrip line incurs large reflection [Fig. 11(f)]. As shown in the Fig. 11(g), the transmission provided by the SSP waveguide over the gap is 9 $\mathrm{dB}$ greater than the microstrip line. Use of the SSP waveguide in regions vulnerable to tear may improve the robustness of the design in wearable applications.

\section{CONClusion}

We have demonstrated conformal propagation and omnidirection radiation of wireless signals emitted from the human body using clothing patterned with surface plasmonic structures. These structures, fabricated using conductive textiles, induce radio-frequency waves emitted by nearby antennas to propagate around the body as surface waves, where they are launched as radiation into the surrounding space. In contrast with prior work on textile-integrated antenna, the surface plasmonic design provides control over propagation and radiation through proximity to the antenna - connectors are not required. Analysis of surface wave excitation using standard waveguide excitation theory allows the amplitude of the excited surface mode to be predicted from the current distribution of the source. Using full-wave simulations and measurements, we verified that such textiles can overcome the obstruction of radiation by the human body and provide nearly omnidirectional angular coverage about 2.62 times larger than in absence of the structures. These results highlight the potential of clothing to engineer the electromagnetic environment around the body in order to enhance the integration of technology into everyday life.

\section{REFERENCES}

[1] S. Han et al., "Battery-free, wireless sensors for full-body pressure and temperature mapping," Sci. Transl. Med., vol. 10, no. 435, p. eaan4950, 2018.

[2] H.-Y. Lin, M. Takahashi, K. Saito, and K. Ito, "Characteristics of electric field and radiation pattern on different locations of the human body for in-body wireless communication," IEEE Trans. Antennas Propag., vol. 61, no. 10 , pp. 5350-5354, Sep. 2013.

[3] C. Mendes and C. Peixeiro, "On-body transmission performance of a novel dual-mode wearable microstrip antenna," IEEE Trans. Antennas Propag., vol. 66, no. 9, pp. 4872-4877, Aug. 2018.

[4] G. A. Conway and W. G. Scanlon, "Antennas for over-body-surface communication at $2.45 \mathrm{GHz}$," IEEE Trans. Antennas Propag., vol. 57, no. 4, pp. 844-855, Mar. 2009.

[5] T. T. Lan, Y. Shinozaki, T. Okura, and H. Arai, "A free-access segmented coplanar waveguide for on-body communication," IEEE Trans. Antennas Propag., vol. 66, no. 9, pp. 4524-4532, 2018.

[6] N. Haga, K. Saito, M. Takahashi, and K. Ito, "Characteristics of cavity slot antenna for body-area networks," IEEE Trans. Antennas Propag., vol. 57, no. 4, pp. 837-843, Mar. 2009.

[7] R. Moro, S. Agneessens, H. Rogier, and M. Bozzi, "Wearable textile antenna in substrate integrated waveguide technology," Electron. Lett., vol. 48, no. 16, pp. 985-987, 2012.

[8] G.-P. Gao, C. Yang, B. Hu, R.-F. Zhang, and S.-F. Wang, "A wearable PIFA with an all-textile metasurface for $5 \mathrm{GHz}$ WBAN applications," IEEE Antennas Wireless Propag. Lett., vol. 18, no. 2, pp. 288-292, Jan. 2019.

[9] C.-H. Lin, K. Saito, M. Takahashi, and K. Ito, "A compact planar inverted-F antenna for $2.45 \mathrm{GHz}$ on-body communications," IEEE Trans. Antennas Propag., vol. 60, no. 9, pp. 4422-4426, 2012. 

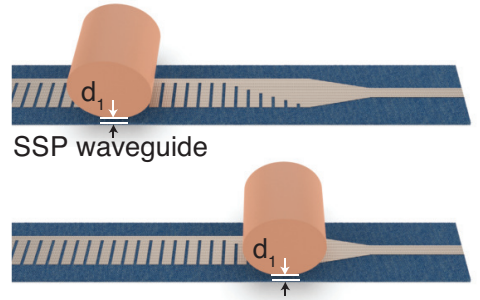

Matching section

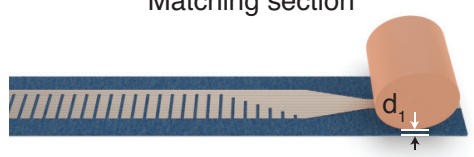

(a)

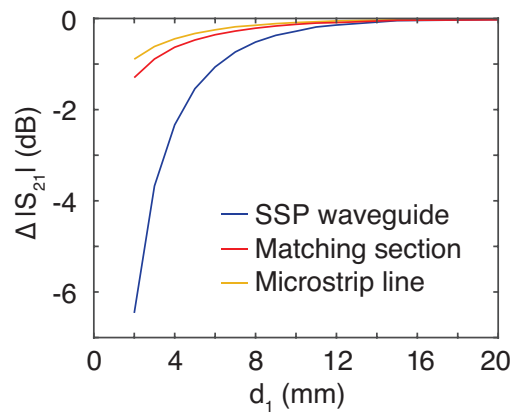

(b)

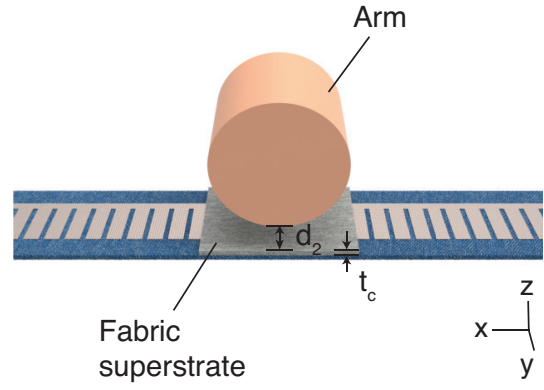

Port 2

Port 1

(c)

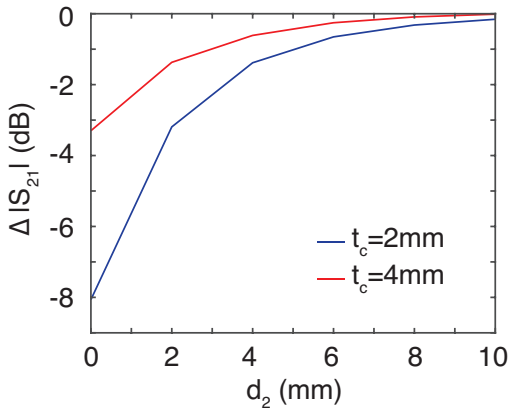

(d)

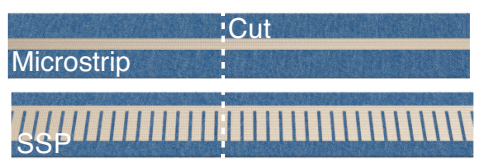

(e)

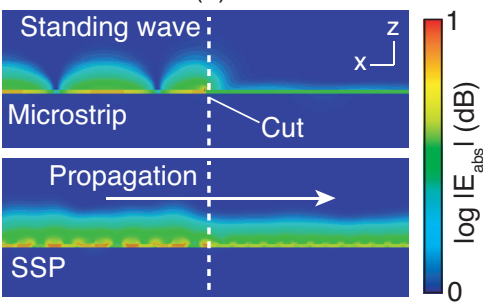

(f)

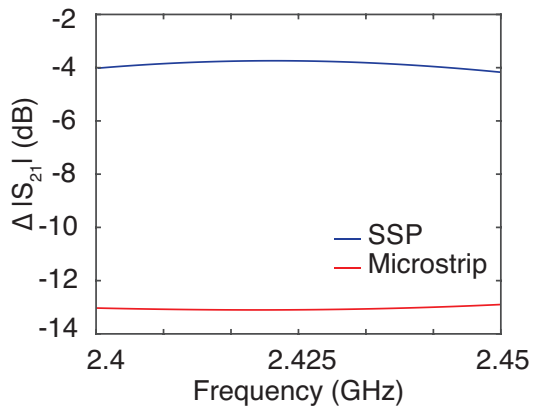

(g)

Fig. 11. Robustness analysis. (a) Illustrations of an environmental object (arm) placed at a distance $d_{1}$ above different sections of the surface plasmonic clothing. (b) Change in transmission $\Delta\left|S_{21}\right|$ (relative to no object) as a function of $d_{1}$ for different positions of the arm. (c) Illustration of a fabric superstrate with thickness of $t_{c}$ added above the SSP waveguide when the arm is placed above the surface of superstrate at a distance $d_{2}$. (d) $\Delta\left|S_{21}\right|$ as a function of $d_{2}$ for different superstrate thickness. (e) Schematics of microstrip line and SSP waveguide cut with a $0.5 \mathrm{~mm}$ gap. (f) $-(\mathrm{g})$ Time-averaged electric field amplitude and $\Delta\left|S_{21}\right|$ after cutting.

[10] S. Bhattacharjee, S. Maity, S. R. B. Chaudhuri, and M. Mitra, "A compact dual-band dual-polarized omnidirectional antenna for on-body applications," IEEE Trans. Antennas Propag., vol. 67, no. 8, pp. 50445053, Aug. 2019.

[11] J. Tak, D.-G. Kang, and J. Choi, "A tapered double cross patch antenna for dual-band monopole-like radiation," Microw. Opt. Technol. Lett., vol. 56, no. 12, pp. 2929-2933, Sep. 2014.

[12] A. Paraskevopoulos, D. de Sousa Fonseca, R. D. Seager, W. G. Whittow, J. C. Vardaxoglou, and A. A. Alexandridis, "Higher-mode textile patch antenna with embroidered vias for on-body communication," IET Microw., Antennas Propag., vol. 10, no. 7, pp. 802-807, 2016.

[13] H. Xiaomu, S. Yan, and G. A. E. Vandenbosch, "Wearable button antenna for dual-band WLAN applications with combined on and offbody radiation patterns," IEEE Trans. Antennas Propag., vol. 65, no. 3, pp. 1384-1387, Mar. 2017.

[14] B. Sanz-Izquierdo, J. Miller, J. C. Batchelor, and M. Sobhy, "Dualband wearable metallic button antennas and transmission in body area networks," IET Microw., Antennas Propag., vol. 4, no. 2, pp. 182-190, 2010.

[15] M. R. Islam and M. Ali, "A $900 \mathrm{MHz}$ beam steering parasitic antenna array for wearable wireless applications," IEEE Trans. Antennas Propag., vol. 61, no. 9, pp. 4520-4527, 2013.

[16] J.-Y. Kim, S.-J. Ha, D. Kim, B. Lee, and C. Jung, "Reconfigurable beam steering antenna using U-slot fabric patch for wrist-wearable applications," J. Electromagn. Waves Appl., vol. 26, no. 11-12, pp. 15451553, 2012.

[17] S.-J. Ha and C. W. Jung, "Reconfigurable beam steering using a microstrip patch antenna with a U-slot for wearable fabric applications," IEEE Antennas Wireless Propag. Lett., vol. 10, pp. 1228-1231, 2011.

[18] A. da Conceicao Andrade, I. P. Fonseca, S. F. Jilani, and A. Alomainy, "Reconfigurable textile-based ultra-wideband antenna for wearable applications," in Proc. 10th Eur. Conf. Antennas Propag. (EuCAP), Apr. 2016, pp. 1-4.

[19] P. B. Samal, P. J. Soh, and G. A. E. Vandenbosch, "UWB all-textile antenna with full ground plane for off-body WBAN communications," IEEE Trans. Antennas Propag., vol. 62, no. 1, pp. 102-108, Dec. 2013.

[20] P. J. Soh, G. A. E. Vandenbosch, S. L. Ooi, and N. H. M. Rais, "Design of a broadband all-textile slotted PIFA," IEEE Trans. Antennas Propag., vol. 60, no. 1, pp. 379-384, Dec. 2011.

[21] P. S. Hall and Y. Hao, "Antennas and propagation for body centric communications," in Proc. Eur. Conf. Antennas Propag. (EuCAP), Jul. 2006, pp. 1-7.

[22] G.-Y. Lee, D. Psychoudakis, C.-C. Chen, and J. L. Volakis, "Omnidirectional vest-mounted body-worn antenna system for UHF operation," IEEE Antennas Wireless Propag. Lett., vol. 10, pp. 581-583, Jan. 2011.

[23] X. Shen, T. J. Cui, D. Martin-Cano, and F. J. Garcia-Vidal, "Conformal surface plasmons propagating on ultrathin and flexible films," Proc. Natl. Acad. Sci. U.S.A., vol. 110, no. 1, pp. 40-45, 2013.

[24] J. Chen et al., "Wideband leaky-wave antennas loaded with gradient metasurface for fixed-beam radiations with customized tilting angles," IEEE Trans. Antennas Propag., vol. 68, no. 1, pp. 161-170, Jan. 2020.

[25] W. Meng, M. H. Feng, T. W. Xuan, S. Shi, and C. T. Jun, "Leaky-wave radiations with arbitrarily customizable polarizations based on spoof surface plasmon polaritons," Phys. Rev. Appl., vol. 12, no. 1, p. 014036, Jul. 2019.

[26] D. Tian, J. Wang, A. Kianinejad, A. Zhang, and Z. N. Chen, "Compact high-efficiency resonator antenna based on dispersion engineering of even-mode spoof surface plasmon polaritons," IEEE Trans. Antennas Propag., vol. 68, no. 4, pp. 2557-2564, Apr. 2020.

[27] M. Wang, S. Sun, H. F. Ma, and T. J. Cui, "Supercompact and ultrawideband surface plasmonic bandpass filter," IEEE Trans. Microw. Theory Tech., vol. 68, no. 2, pp. 732-740, Feb. 2020.

[28] X. Gao, L. Zhou, Z. Liao, H. F. Ma, and T. J. Cui, "An ultra-wideband surface plasmonic filter in microwave frequency," Appl. Phys. Lett., vol. 104, no. 19, p. 191603, 2014.

[29] Y. J. Zhou, Q. X. Xiao, and B. J. Yang, "Spoof localized surface plasmons on ultrathin textured MIM ring resonator with enhanced resonances," Sci. Rep., vol. 5, p. 14819, 2015. 
[30] S. Zhou et al., "Spoof surface plasmon polaritons power divider with large isolation," Sci. Rep., vol. 8, no. 1, p. 5947, 2018.

[31] X. Tian et al., "Wireless body sensor networks based on metamaterial textiles," Nat. Electron., vol. 2, pp. 335-342, Jun. 2019.

[32] S. A. Maier, Plasmonics: Fundamentals and Applications. Springer: New York, NY, 2007.

[33] X. Wan and T. J. Cui, "Guiding spoof surface plasmon polaritons by infinitely thin grooved metal strip," AIP Adv., vol. 4, no. 4, p. 047137 , 2014.

[34] J. D. Jackson, Classical Electrodynamics. Hoboken, NJ: John Wiley \& Sons, 1999.

[35] X.-F. Zhang, J. Fan, and J.-X. Chen, "High gain and high-efficiency millimeter-wave antenna based on spoof surface plasmon polaritons," IEEE Trans. Antennas Propag., vol. 67, no. 1, pp. 687-691, Jan. 2019.

[36] X. Du, H. Li, and Y. Yin, "Wideband fish-bone antenna utilizing oddmode spoof surface plasmon polaritons for endfire radiation," IEEE Trans. Antennas Propag., vol. 67, no. 7, pp. 4848-4853, 2019.

[37] A. Kianinejad, Z. N. Chen, and C.-W. Qiu, "Low-loss spoof surface plasmon slow-wave transmission lines with compact transition and high isolation," IEEE Trans. Microw. Theory Tech., vol. 64, no. 10, pp. 30783086, Sep. 2016.

[38] W. Zhang, G. Zhu, L. Sun, and F. Lin, "Trapping of surface plasmon wave through gradient corrugated strip with underlayer ground and manipulating its propagation," Appl. Phys. Lett., vol. 106, no. 2, pp. 021 104-6, Jan. 2015

[39] H. F. Ma, X. Shen, Q. Cheng, W. X. Jiang, and T. J. Cui, "Broadband and high-efficiency conversion from guided waves to spoof surface plasmon polaritons," Laser Photonics Rev., vol. 8, no. 1, pp. 146-151, Nov. 2013.

[40] R. Salvado, C. Loss, R. Gonçalves, and P. Pinho, "Textile materials for the design of wearable antennas: A survey," Sensors, vol. 12, no. 11, pp. 15 841-15 857, 2012.

[41] A. Dabbagh, B. J. J. Abdullah, C. Ramasindarum, and N. H. Abu Kasim, "Tissue-mimicking gel phantoms for thermal therapy studies," Ultrasonic Imaging, vol. 36, no. 4, pp. 291-316, Jun. 2014. 